\title{
A Young Girl of 12 Year with Pancreatoblastoma; a Case Report and Short Discussion
}

\author{
Israt Jahan ${ }^{1 *}$, Sarwar Alam², Md. Abdul Bari ${ }^{3}$, Tarek $\operatorname{Hasan}^{4}$, Md Niaz Mostafa
}

${ }^{1}$ Resident Department of Oncology, ${ }^{2}$ Profssor and head Dept of Oncology BSMMU, ${ }^{3}$ Associate Professor Dept of Oncology BSMMU, ${ }^{4}$ Resident Dept of Oncology BSMMU, ${ }^{5}$ Resident Dept of Pathology BSMMU, Bangabandhu Sheikh Mujib Medical University (BSMMU), Shahbagh, Dhaka

DOI: $10.36347 /$ simcr.2020.v08i03.045

| Received: 16.03.2020 | Accepted: 23.03.2020 | Published: 30.03.2020

*Corresponding author: Israt Jahan, MBBS

Abstract

A 12 years old girl admitted in Bangabandhu Sheikh Mujib Medical University (BSMMU) with the complaints of occasional abdominal pain, vomiting and a mass in abdomen for four months. Her physical examination revealed a large solid mass measuring about $10 \times 8 \mathrm{~cm}$ in left hypochondriac and epigastric region, CT scan of abdomen revealed mass was pancreatic in origin. Surgery was done and during exploration the mass was nodular, capsulated and cut surface was solid with necrotic and haemorrhagic areas, histopathology report was pancreatoblastoma and there was capsular and perineural invasion. She completed adjuvant chemotherapy and was on follow up but after two years she died due to local recurrence. Pancreatoblastoma (PB) is a rare childhood malignancy of pancreatic islet cells and so far our knowledge goes it is one of the few cases diagnosed in Bangladesh. In most cases tumor is located in the head of the pancreas and patients usually presents with abdominal pain and mass, vomiting, diarrhea or jaundice. The presenting features are non-specific, so it is difficult to distinguish from other inra-abdominal tumors. Here, we describe the diagnosis, clinical and histological characteristics of the tumor, and the management.

Keywords: Pancreatoblastoma, children, acinar cell, histopathology, chemotherapy.

Copyright @ 2020: This is an open-access article distributed under the terms of the Creative Commons Attribution license which permits unrestricted use, distribution, and reproduction in any medium for non-commercial use (NonCommercial, or CC-BY-NC) provided the original author and source are credited.

\section{INTRODUCTION}

Pancreatoblastoma (PB), or infantile pancreatic carcinoma, is an extremely rare pancreatic tumor in children, comprising $0.5 \%$ of pancreatic non-endocrine tumors [1]; <100 cases have been reported in the literature [2]. The clinical presentations of these tumors are varied. They can present with abdominal pain, abdominal mass, diarrhoea or upper gastrointestinal bleeding. Most of the time, they are asymptomatic. The presenting features are highly non-specific and this leads to diagnostic dilemmas. Ultrasound and computed tomography (CT) scan may be useful but preoperative diagnosis is often quite difficult. Surgery is the most optimal treatment for pancreatoblastoma. Prognosis of this rare tumor is good, when the tumor is resected completely. Prognosis is poorer, when there is metastasis or when the tumour is inoperable

\section{Case Report}

Our patient, a 12 years old girl admitted to paediatric surgery department of Bangabandhu Sheikh Mujib Medical University (BSMMU) with the complaints of occasional abdominal pain for four months and swelling in upper abdomen for one month.
The pain was dull aching and confined to left epigastric region, there was no relieving or aggravating factor and pain increased in severity for one month. The swelling was firm in consistency, non-tender, gradually increasing in size. She had $\mathrm{H} / \mathrm{O}$ occasional vomiting but no history of diarrhoea, fever or jaundice. On admission her vital signs were normal .Per abdominal examination revealed a lump on left epigastric and hypochondriac region measuring about $10 \times 8 \mathrm{~cm}$, firm in consistency, non-tender with restricted movement on side to side. Her blood reports was normal except liver enzymes was elevated and serum alpha Feto protein was $101 \mathrm{ng} / \mathrm{ml}$. CT scan of whole abdomen was done which revealed a $14 \times 8 \mathrm{~cm}$ heterogeneously enhancing mixed solid and cystic mixed density mass arises from body and tail of the pancreas without arterial encasement or locoregional lymphadenopathy, pancreatic duct was mildly dilated.

The patient underwent laparotomy and a large mass measuring about $12 \times 10 \mathrm{~cm}$ was identified on the pancreas, numerous dilated blood vessels was seen above the mass and the mass was adherent to transverse mesocolon and liver, but peritoneum was normal. Distal pancreatectomy with splenectomy was done and the 
mass was sent for histopathological examination. Her post-operative period was uneventful.

Histopathology report revealed a tumor of solid sheets of uniform tumor cells with partly acinar and ductal differentiation. Areas of necrosis are present .Capsular, vascular and perineural invasion is seen. Resected lymph nodes were free of tumor.

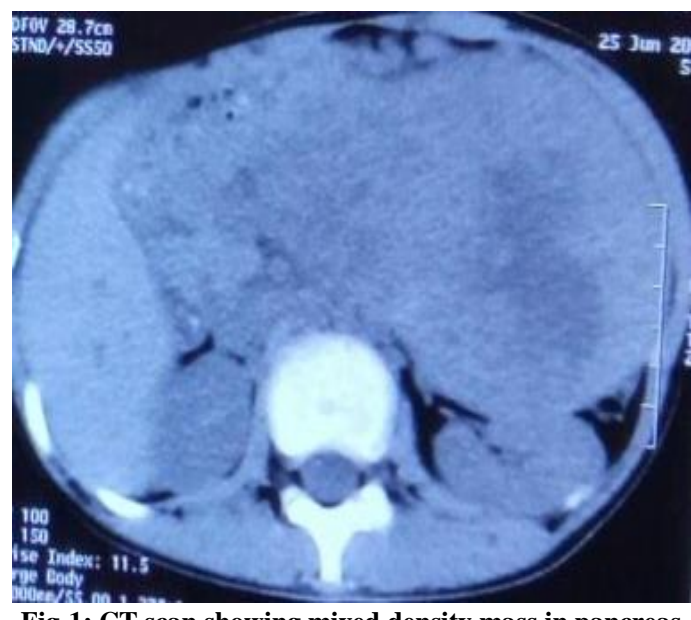

Fig-1: CT scan showing mixed density mass in pancreas

After surgery patient was referred to Oncology Department of BSMMU for further management and considering her per operative findings and histopathology report patient was advised for adjuvant chemotherapy with 5 flurouracil and leucovorin and patient has completed 6 cycles of chemotherapy without any complications. Patient was on regular follow up but unfortunately after 2 years there was locoregional recurrence and patient was offered surgery, but her parents refused to take further treatment and patient died thereafter.

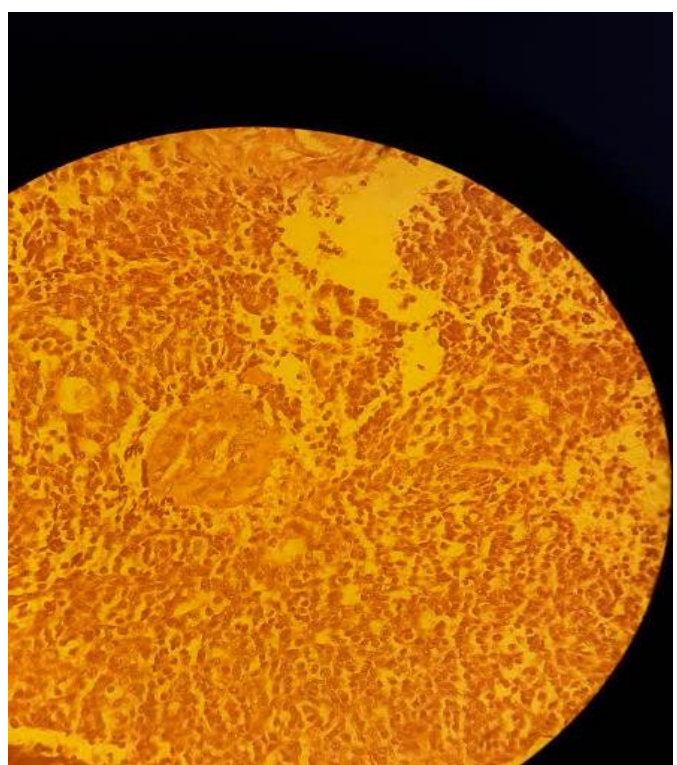

Fig-2: Histopathology showing tumor cells with partly acinar and ductal differentiation

\section{DISCUSSION}

Pancreatoblastoma is a rare pancreatic tumour of acinar cell origin. Reportedly, most of pancreatoblastomas have been found in children less than 8 years old, but the entity also occurs very rarely in adulthood [4]. Highest incidence is found in the first decade of life with a predilection for males and Asians [5]. Pancreatoblastoma was first described by Becker [6]. In 1957, which was termed "infantile pancreatic carcinoma [3]"'? The tumour's histology resembled fetal pancreatic tissue at approximately 7 weeks' gestation as reported by Horie[7] in 1977, to account for the histologic features of this tumour and suggested the term pancreatoblastoma.

A pancreatoblastoma is a slow growing tumor, soft and well circumscribed, usually very large in size at the time of diagnosis. ${ }^{1-}$ Approximately half of the tumors are found in the pancreatic head. ${ }^{10}$ Macroscopically, most pancreatoblastomas are encapsulated and exhibit intratumoral hemorrhage and necrosis; cystic degeneration and calcifications can also be found[10]. The tumor is histologically characterized by different types of epithelial neoplastic cells arranged in lobules separated by gross fibrous stroma [7]. Solid sheets of neoplastic cells with an acinar and squamous like pattern are evident along with nuclei of keratinization, the so-called "squamoid corpuscles", which represent the most typical aspect of pancreatoblastoma; immunohistochemistry may show positivity for acinar, ductal and endocrine differentiation [4]. Tumors with overlapping features between pancreatoblastoma and neoplasms with mixed endocrine and solidpseudopapillary differentiation have rarely been noted [11]. Incidental detection of an abdominal mass is the most frequent clinical presentation of pancreatoblastomas; symptomatic patients usually complain of abdominal pain, weight loss, diarrhea and vomiting; instead, jaundice is rare [9]. Most of the tumors are grossly inhomogeneous with all diagnostic imaging modalities due to coexisting solid and cystic areas; septations and calcifications can also be present. $\frac{5}{}$, Despite the fact that radiological findings may suggest an encapsulated tumor, local infiltration cannot be excluded preoperatively. In pediatric patients, very large pancreatoblastomas cannot be differentiated radiologically from hepatoblastoma or other infantile tumors [9]. Treatment of pancreatoblastoma is surgery. Although the surgical approach for PB is not standardized, total resection is so far the only choice for treating these patients [8]. The role of postoperative chemotherapy and appropriate regimen is uncertain. Chemotherapy has been used for unresectable tumors, to treat metastatic disease or in the adjuvant setting. 5Flurouracil, cyclophosphamide, actinomycin D, vincristine, cisplatin, doxorubicin, ifosfamide and gemcitabine alone or in combination, have been used with very modest results [2]. Our patient had tumor on tail of the pancreas and it had lymphatic, vascular and perinural invasion, so postoperative chemotherapy was 
considered with affordable schedule. She has completed chemo uneventfully but unfortunately after two years she developed local recurrence and died.

\section{CONCLUSION}

Pancreatoblastoma is a rare pancreatic tumor in childhood, an early resection of the tumor with postoperative chemotherapy is the key to the good outcome. Prognosis is poor when there is metastasis or when the tumor is inoperable.

\section{REFERENCES}

1. Saif MW. Pancreatoblastoma. JOP: Journal of the pancreas. 2007;8(1):55-63.

2. Halperin EC, Wazer DE,Perez CA, Brady LW. Principles and Practice of Radiation Oncology.2013;1717.

3. Tao C, Tang DX, LI MJ Pancreatoblastoma: A Case Report and Literature Review HK J Paediatr (new series). 2010;15:324-326

4. Klimstra DS, Wenig BM, Adair CF, Heffess CS. Pancreatoblastoma. A clinicopathologic study and review of the literature. Am J Surg Pathol. 1995;19:1371-89.
5. Chung EM, Travis MD, Conran RM. Pancreatic tumors in children: radiologic-pathologic correlation. Radiographics. 2006;26:1211-38.

6. Becker WF. Pancreatoduodenectomy for carcinoma of the pancreas in an infant; report of a case. Ann Surg 1957;145:864-70.

7. Horie A, Yano Y, Kotoo Y, Miwa A. Morphogenesis of pancreatoblastoma, infantile carcinoma of the pancreas: Report of two cases. Cancer. 1977;39:247-54.

8. Dumitrascu T, Stanciulea O, Herlea V, Tomulescu $\mathrm{V}$, Ionescu M. Central pancreatectomy for pancreatoblastoma in a 16-year-old girl. J Pediatric Surg. 2011;46:17-21.

9. Montemarano H, Lonergan GJ, Bulas DI, Selby DM. Pancreatoblastoma: imaging findings in 10 patients and review of the literature. Radiology. 2000; 214:476-82.

10. Kohda E, Iseki M, Ikawa H, Endoh M, Yokoyama J, Mukai M, Hata J, Yamazaki H, Miyauchi J, Saeki M. Pancreatoblastoma: three original cases and review of the literature. Acta Radiologica. $2000 \mathrm{Jul}$;41(4):334-7.

11. Ohike N, Yamochi T, Shiokawa A, Yoshida T, Yamazaki T, Date Y, Morohoshi T. A peculiar variant of pancreatoblastoma in an adult. Pancreas. 2008 Apr 1;36(3):320-2. 